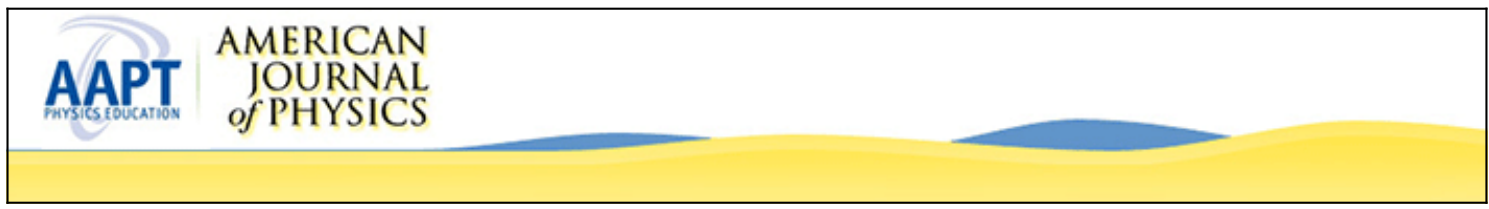

\title{
On the Classical Model of Nuclear Fission
}

M. S. Plesset

Citation: American Journal of Physics 9, 1 (1941); doi: 10.1119/1.1991623

View online: http://dx.doi.org/10.1119/1.1991623

View Table of Contents: http://scitation.aip.org/content/aapt/journal/ajp/9/1?ver=pdfcov

Published by the American Association of Physics Teachers

\section{Articles you may be interested in}

Nuclear fission of $\mathrm{Fm}$ isotopes

AIP Conf. Proc. 1238, 325 (2010); 10.1063/1.3455960

Numerical Modeling of Scission Neutrons Emitted During Nuclear Fission

AIP Conf. Proc. 972, 526 (2008); 10.1063/1.2870440

Stochastic Model of the Tilting Mode in Nuclear Fission

AIP Conf. Proc. 704, 130 (2004); 10.1063/1.1737104

Delayed nuclear fission

Phys. Part. Nucl. 30, 666 (1999); 10.1134/1.953123

Discovery of Nuclear Fission

Am. J. Phys. 32, 9 (1964); 10.1119/1.1970127

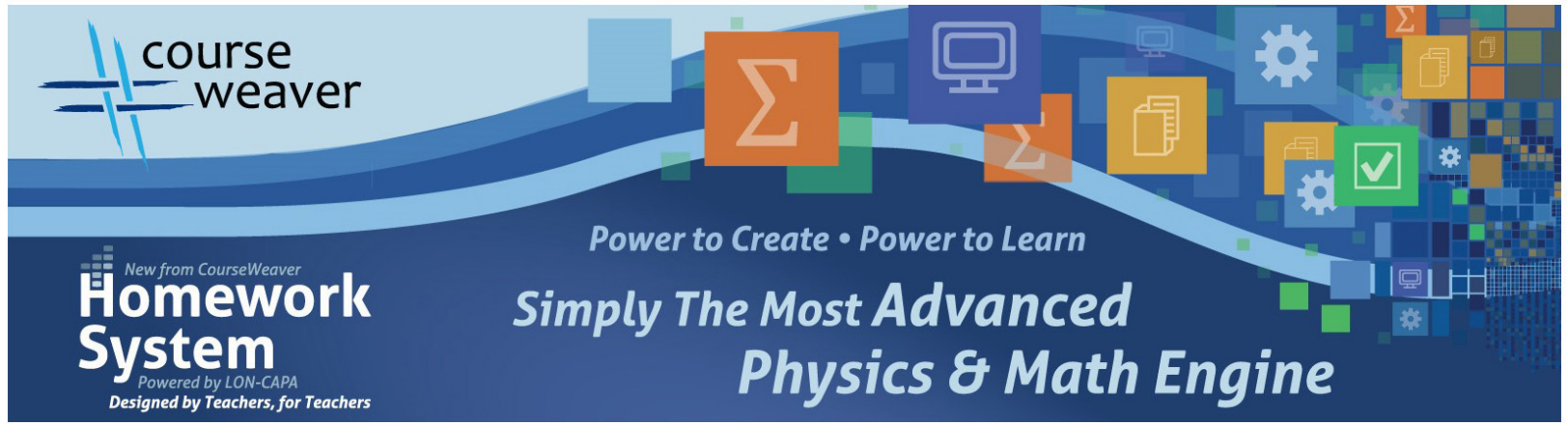




\title{
AMERICAN JOURNAL of PHYSICS
}

(Formerly The American Physics Teacher)

A Journal Devoted to the Instructional and Cultural Aspects of Physical Science

\begin{tabular}{lll}
\hline Volume 9 & FEBRUARY, 1941 & NUMBER 1 \\
\hline
\end{tabular}

\section{On the Classical Model of Nuclear Fission}

\author{
M. S. Plesset \\ Norman Bridge Laboratory of Physics, California Institute of Technology, Pasadena, California
}

1.

$\mathrm{T}$ HE first experiments on neutron bombardment of various elements carried out by Fermi and his collaborators ${ }^{1}$ included the study of the group of activities observed in uranium which were at that time ascribed to transuranic elements. The great number of studies ${ }^{2}$ following this first work led finally to the results of Hahn and Strassmann ${ }^{3}$ which showed clearly that many of the activities ascribed to transuranic elements came, instead, from nuclei of approximately half the mass of uranium. The startling conclusion that these activities must arise from the splitting of the uranium nucleus under neutron bombardment into two fragment nuclei was pointed out by Meitner and Frisch, ${ }^{4}$ and was quickly confirmed by subsequent experiments. In the first theoretical discussion of this new type of nuclear reaction, Meitner and Frisch ${ }^{4}$ proposed the name fission for the process, and compared it with the splitting that may take place in a liquid drop in oscillation. This model was supported by $\mathrm{Bohr}^{5}$ who correlated it with other nuclear

\footnotetext{
1 E. Fermi, Nature 133, 898 (1934); Fermi, Amaldi, D'Agostino, Rasetti and Segre, Proc. Roy. Soc. A146, 483 (1934).

${ }^{2}$ Cf. the review article by L. A. Turner, Rev. Mod. Phys. 12, 1 (1940).

${ }^{3}$ O. Hahn and F. Strassmann, Naturwiss. 27, 11 (1939); 27,89 (1939).

${ }^{4}$ L. Meitner and O. R. Frisch, Nature 143, 239 (1939); 143, 471 (1939).

${ }^{5}$ N. Bohr, Nature 143, 330 (1939); Phys. Rev. 55, 418 (1939).
}

properties and, at the same time, emphasized how far the phenomenon of nuclear fission may be described classically. A very complete theoretical discussion of both the classical and quantum aspects of fission was given by Bohr and Wheeler, ${ }^{6}$ and it is proposed here to describe some of the classical theory of fission developed by these authors.

$$
2
$$

In this section qualitative arguments from general properties of nuclear forces will be given to justify the classical model used to picture fission. This model is more accurate for the heavier nuclei, but these are the only ones of actual interest because only in the heaviest nuclei is the fission process experimentally accessible.

In the first place, the forces between nuclear particles, neutrons and protons, are known to be of short range; that is, of the order of $2 \times 10^{-13}$ $\mathrm{cm}$. A heavy nucleus, such as uranium, has a diameter of the order of $18 \times 10^{-13} \mathrm{~cm}$. Thus, it may be said that a heavy nucleus has a fairly welldefined surface. Of course, from a macroscopic point of view, the surface of a nucleus is poorly defined compared with that, for example, of a water droplet, in which the size of the droplet and the range of the forces between component molecules are of entirely different orders of magnitude.

${ }^{6}$ N. Bohr and J. A. Wheeler, Phys. Rev. 56, 426 (1939). 
The second general property of nuclear forces that will be used here is inferred from the following observation: The most important contribution to the binding energy of nuclei increases linearly with the number of component nuclear particles $A$. If the attractions between nuclear particles were of the type familiar from classical theory-that is, if each particle in the nucleus attracted every other particle in the nucleus-the binding energy would vary as $A^{2}$. The simplest interpretation of the observed variation is that there is a saturation of nuclear forces. This saturation may be pictured as follows: A neutron most strongly attracts another neutron that is most nearly in the same quantum state (except for spin) as the given neutron; a similar law is considered to hold between neutrons and protons and, aside from their electrostatic repulsion by the Coulomb law, between protons and protons. $^{7}$ Saturation arises then in these interactions because the Pauli exclusion principle limits to two the number of particles of a given kind that may be in the same state. There is thus an approximate pairing of neutron-proton interactions with each particle of one kind strongly attracting at most one particle of the same kind, and at most two particles of the second kind. On this basis, one may understand the difference in neutron binding energy between an isotope with an even number of neutrons and one with an odd number. For example, the odd neutron-number uranium isotope ${ }_{92} \mathrm{U}^{235}$ upon capturing an additional neutron of low energy has greater energy of excitation than the even neutron-number isotope ${ }_{92} \mathrm{U}^{238}$ upon similar capture; Bohr and Wheeler estimate these excitation energies at $6.4 \mathrm{Mev}$ and $5.2 \mathrm{Mev}$, respectively.

Saturation implies not only that the binding energy is proportional to the number of particles but also that nuclear matter has constant density from nucleus to nucleus and in a given nucleus. Thus, in a nucleus of radius $R$, the average number of particles per unit volume is $\delta=$ const. $=A /(4 \pi / 3) R^{3}$. Consequently, one should have $R \propto A^{\frac{1}{2}}=r_{0} A^{\frac{1}{3}}$. This dependence of $R$ on the number of component nuclear particles $A$ is

\footnotetext{
${ }^{7}$ This picture is inaccurate inasmuch as it is not found possible to assign a state to each particle independently of the remaining particles. A more correct description leads to similar qualitative conclusions.
}

verified experimentally, and $r_{0}$ is found to be approximately $1.48 \times 10^{-13} \mathrm{~cm}$. The total nuclear chatge $Z e$ also is taken to be uniformly distributed throughout the nuclear volume, so that the electrostatic energy of the nucleus is that of a uniformly charged sphere of the radius $R$, namely, $(3 / 5)(Z e)^{2} / R$. It is reasonable to assume that there should be no appreciable tendency for the protons to concentrate near the nuclear boundary since nuclear attractions are larger than the electrostatic repulsion operating between protons.

At the boundary of a nucleus there occurs a special effect, namely, the desaturation of the nuclear short range binding forces. This desaturation may be described by saying that the surface of the nucleus is under tension in analogy with the desaturation taking place at the boundary of a liquid drop which is similarly described by a surface tension. Because of the constant volume density of nuclei, any deformation of a nucleus must be considered as taking place at constant volume. Consequently, the energy of the nuclear binding forces acting in the volume of the nucleus remains constant with change of shape, and the only changes of energy which need be considered are in the surface energy and in the electrostatic energy. In summary, it may be said that the approximate model taken for a heavy nucleus is a uniformly charged, incompressible, fluid sphere under surface tension.

To get an idea of the orders of magnitude of the energies mentioned, one may refer to an approximate, "semiempirical" formula ${ }^{8}$ for the total binding energy of a nucleus of mass $M$ containing $N$ neutrons, each of mass $M_{n}$, and $Z$ protons, each of mass $M_{p}$,

$$
\begin{aligned}
N M_{n}+Z M_{p}-M & =\alpha A-4 \pi r_{0}^{2} A^{\frac{2}{3}} O \\
& -(3 / 5)(Z e)^{2} / r_{0} A^{\frac{3}{3}}-\beta(N-Z)^{2} / A .
\end{aligned}
$$

The first term in the right-hand member of Eq. (1) is the main contribution to the binding energy and arises from the saturation or volume forces; the second term represents a decrease in stability caused by surface desaturation, or "surface tension"; the third term is a decrease in stability arising from Coulomb repulsion; and the last term is a decrease in stability arising from an

\footnotetext{
${ }^{8}$ H. A. Bethe and R. F. Bacher, Rev. Mod. Phys. 8,
} 165 (1936). 


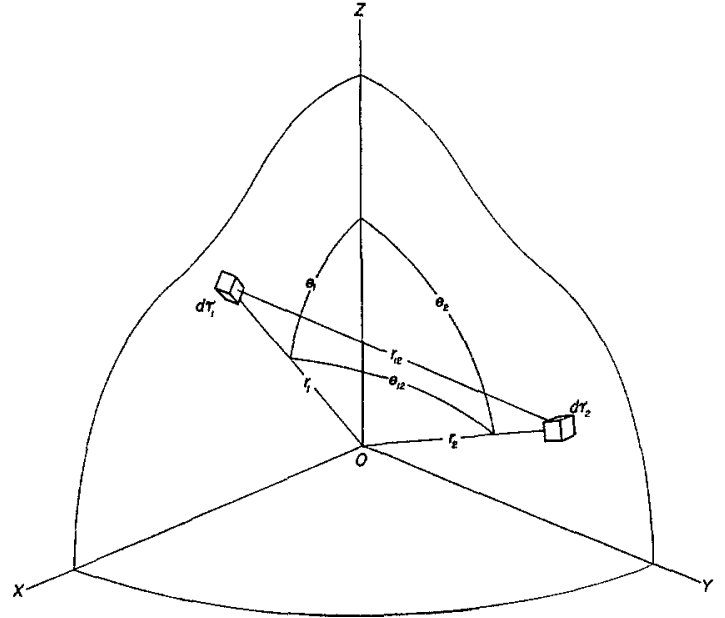

FIG. 1. Coordinates used in the calculation of nuclear electrostatic energy.

excess in the number of neutrons over the number of protons. For ${ }_{92} \mathrm{U}^{239}, \alpha A \approx 3300 \mathrm{Mev} ; 4 \pi R^{2} O$ $=4 \pi \gamma_{0}{ }^{2} A^{\frac{2}{3}} O \approx 540 \mathrm{Mev} ;(3 / 5)(Z e)^{2} / R \approx 800 \mathrm{Mev} ;$ $\beta(N-Z)^{2} / A \approx 250 \mathrm{Mev}$.

If a nucleus of mass $M$ splits into smaller parts of masses $M_{1}, M_{2}, \cdots$, the total energy released is, according to the relativistic mass-energy formula, $E=M c^{2}-\sum M_{i} c^{2}$, where the original nucleus and the resulting nuclei are unexcited and at rest. The splitting of a heavy nucleus into, for example, two fragments of approximately the same size leads to an abnormally high mass-tocharge ratio so that the mass energies of the fragments lie outside the ordinary range which has been measured directly. The excess neutron content of the fragments will be reduced by $\beta$ emission and some neutron emission. A detailed estimate ${ }^{6}$ of the mass energy of such fragments as would arise from fission leads to the conclusion that splitting is exothermic for nuclei with values of $A$ larger than about 100 ; for uranium the energy released from division into two nearly equal fragments is, on the basis of this estimate, of the order of $200 \mathrm{Mev}^{9}$

A very rough estimate of the fission energy release may be made using $\mathrm{Eq}$. (1). The values of the constants in this equation are taken to be: $\alpha=13.9 \mathrm{Mev} ; 4 \pi r_{0}{ }^{2} 0=14 \mathrm{Mev}$; $(3 / 5) e^{2} / r_{0}=0.59 \mathrm{Mev} ; \beta=19.5 \mathrm{Mev}$. If one assumes the fission reaction

$$
{ }_{92} \mathrm{U}^{239} \rightarrow{ }_{46} \mathrm{Pd}^{119}+{ }_{46} \mathrm{Pd}^{120},
$$

and calculates by Eq. (1) the difference between the initial and final mass energies for this case, the terms in $\alpha$ and $\beta$ cancel. The energy release $\Delta E$ is then the difference between the initial and final values for the surface and Coulomb energies,

$$
\begin{aligned}
E=4 \pi r_{0}{ }^{2} A^{3} O-2 \cdot 4 \pi r_{0}{ }^{2}(A / 2)^{2} O & +(3 / 5)\left(e^{2} / r_{0}\right) Z^{2} / A^{\frac{1}{3}} \\
& -2(3 / 5)\left(e^{2} / r_{0}\right)(Z / 2)^{2} /(A / 2)^{\frac{1}{3}},
\end{aligned}
$$

which is here $\sim 160 \mathrm{Mev}$. The nuclei ${ }_{46} \mathrm{Pd}^{119}$ and ${ }_{46} \mathrm{Pd}^{120}$ would be unstable because of their excess neutron content and would presumably go over into the stable nuclei ${ }_{50} \mathrm{Sn}^{119}$ and ${ }_{50} \mathrm{Sn}^{120}$, respectively. The mass energy difference between ${ }_{92} \mathrm{U}^{239}$ and the latter nuclei gives from Eq. (1) the same surface energy loss as before, a slightly smaller Coulomb energy gain, and a gain from the terms in $\beta$ with a resulting total energy release in the fission to the final products of the order of $180 \mathrm{Mev}$.

If the values of the nuclear constants assumed here are used, it will be shown in Sec. 3 that, while fission is energetically possible, all the nuclei, including the uranium isotopes, are stable when unexcited: This stability persists even in the nuclei with the largest values of $Z$ because the decrease in electrostatic energy in a small deformation from the spherical shape is overcompensated by the increase in surface energy arising from the increase in exposed nuclear surface.

3.

The deformation of a nucleus initially a sphere of radius $R$ may be specified by giving the magnitude of the radius vector from the center of mass to the boundary. If the deformed nucleus is taken to have zonal symmetry - that is, if its boundary is a surface of revolution-this radius vector will be independent of the azimuthal angle $\varphi$, and will be determined only by the colatitude $\theta$. In this case the radius vector to the boundary, $r(\theta)$, may be expressed as a sum of Legendre polynomials,

$$
\begin{aligned}
r(\theta) & =R\left\{1+\alpha_{0}+\alpha_{1} P_{1}(\cos \theta)+\alpha_{2} P_{2}(\cos \theta)+\cdots\right\} \\
& =R\left\{1+\alpha_{0}+Q(\cos \theta)\right\} .
\end{aligned}
$$

\footnotetext{
${ }^{9}$ Experimental value measured by M. C. Henderson, Phys. Rev. [58, $\left.774(1940)\right]$ is $\sim 180$ Mev. See also M. H. Kanner and H. H. Barschall, Phys. Rev. 57, 372 (1940).
} 
The Legendre polynomials have their usual values so that

$$
\int_{0}^{\pi} P_{n}^{2}(\cos \theta) \sin \theta \mathrm{d} \theta=2 /(2 n+1) ; \quad \int_{0}^{\pi} P_{m}(\cos \theta) P_{n}(\cos \theta) \sin \theta \mathrm{d} \theta=0,(n \neq m) .
$$

For stability considerations, the energy need be calculated only for small deformations, and terms containing $\alpha$ 's with powers higher than the second will be neglected. In this approximation, the limitation of zonal symmetry in the deformation is unessential; the most general deformation, which would be expressed in terms of surface harmonics, leads to the same energy expressions. The requirement that the volume be unchanged determines $\alpha_{0}$ :

$$
4 \pi R^{3} / 3=\int_{0}^{2 \pi} \mathrm{d} \varphi \int_{0}^{\pi} \sin \theta \mathrm{d} \theta \int_{0}^{r(\theta)} r^{2} \mathrm{~d} r
$$

from which one finds in the present approximation,

$$
\alpha_{0}=-\sum_{n=1} \alpha_{n}^{2} /(2 n+1)=-K
$$

The surface energy $E_{S}$ of the deformed nucleus is given by the product of the surface tension $O$ and the total area, or

$$
E_{S}=O \int \mathrm{d} S
$$

where, since $r(\theta)$ is independent of $\varphi$,

$$
\begin{aligned}
\mathrm{d} S & =r^{2}(\theta) \sin \theta \mathrm{d} \theta \mathrm{d} \varphi\left[1+(\mathrm{d} r / r \mathrm{~d} \theta)^{2}\right]^{\frac{1}{3}}, \\
& \approx r^{2}(\theta) \sin \theta \mathrm{d} \theta \mathrm{d} \varphi+\left(\frac{1}{2}\right)(\mathrm{d} r / \mathrm{d} \theta)^{2} \sin \theta \mathrm{d} \theta \mathrm{d} \varphi+\cdots
\end{aligned}
$$

One may separate $E_{S}$ into two terms, $E_{S}^{\prime}$ and $E^{\prime \prime}{ }_{S}$, corresponding to these two terms in Eq. (5) for $\mathrm{d} S$. Then

For the second term, one has

$$
\begin{aligned}
E^{\prime}{ }_{S} & =2 \pi R^{2} O \int_{0}^{\pi}\left(1+\alpha_{0}+Q\right)^{2} \sin \theta \mathrm{d} \theta \\
& \approx 4 \pi R^{2} O\left\{\left(1+\alpha_{0}\right)^{2}+K\right\} \\
& \approx 4 \pi R^{2} O(1-K) .
\end{aligned}
$$

$$
E^{\prime \prime}{ }_{S}=\pi R^{2} O \int_{0}^{\pi}\left\{\sum_{n=1} \alpha_{n}{ }^{2}\left(\mathrm{~d} P_{n} / \mathrm{d} \theta\right)^{2}+\sum_{n \neq m} \alpha_{n} \alpha_{m}\left(\mathrm{~d} P_{n} / \mathrm{d} \theta\right)\left(\mathrm{d} P_{m} / \mathrm{d} \theta\right)\right\} \sin \theta \mathrm{d} \theta .
$$

Since $\mathrm{d} P_{n} / \mathrm{d} \theta=-\sin \theta \mathrm{d} P_{n} / \mathrm{d}(\cos \theta)$, and since

one has

$$
\int_{0}^{\pi}\left(\mathrm{d} P_{n} / \mathrm{d}(\cos \theta)\right)\left(\mathrm{d} P_{m} / \mathrm{d}(\cos \theta)\right) \sin ^{3} \theta \mathrm{d} \theta=n(n+1) \int_{0}^{\pi} P_{n}(\cos \theta) P_{m}(\cos \theta) \sin \theta \mathrm{d} \theta,
$$

Thus,

$$
E_{S}^{\prime \prime}=2 \pi R^{2} O \sum_{n=1} \alpha_{n}^{2} n(n+1) /(2 n+1)
$$

$$
E_{S}=4 \pi R^{2} O\left\{1+\sum_{n=1} \alpha_{n}^{2}(n-1)(n+2) / 2(2 n+1)\right\} .
$$

The electrostatic energy $E_{E}$ may also be determined by a straightforward calculation..$^{10}$ As the

${ }^{10}$ For a computation of the surface and electrostatic energies along somewhat different lines, see J. Frenkel, J. Phys. U.S.S.R. 1, 125 (1939). 
electric charge density $\rho=Z e /\left(4 \pi R^{8} / 3\right)$ is constant, one may write

$$
E_{E}=\frac{1}{2} \rho^{2} \iint \mathrm{d} \tau_{1} \mathrm{~d} \tau_{2} / r_{12},
$$

where $\mathrm{d} \tau_{1}$ and $\mathrm{d} \tau_{2}$ are elements of volume at the points $\left(r_{1}, \theta_{1}, \varphi_{1}\right),\left(r_{2}, \theta_{2}, \varphi_{2}\right)$ within the nucleus, and $r_{12}$ is the distance between them (Fig. 1). If the electrostatic potential of the charge distribution at an interior point $\left(r_{1}, \theta_{1}, \varphi_{1}\right)$ is denoted by $\rho V\left(r_{1}, \theta_{1}\right)$, then

and

$$
V\left(r_{1}, \theta_{1}\right)=\int \mathrm{d} \tau_{2} / r_{12}
$$

$$
E_{E} / \frac{1}{2} \rho^{2}=\int V\left(r_{1}, \theta_{1}\right) \mathrm{d} \tau_{1}
$$

To calculate $V\left(r_{1}, \theta_{1}\right)$, one may use the following expressions for $1 / r_{12}$ :

$$
\begin{aligned}
1 / r_{12} & =\sum_{k=0}^{\infty}\left(r_{2}^{k} / r_{1}^{k+1}\right) P_{k}\left(\cos \theta_{12}\right), \quad r_{2}<r_{1} ; \\
& =\sum_{k=0}^{\infty}\left(r_{1}^{k} / r_{2}^{k+1}\right) P_{k}\left(\cos \theta_{12}\right), \quad r_{2}>r_{1} .
\end{aligned}
$$

Then,

$$
V\left(r_{1}, \theta_{1}\right)=\iint \sin \theta_{2} \mathrm{~d} \theta_{2} \mathrm{~d} \varphi_{2} \int_{0}^{r_{1}} r_{2}^{2} \mathrm{~d} r_{2} / r_{12}+\iint \sin \theta_{2} \mathrm{~d} \theta_{2} \mathrm{~d} \varphi_{2} \int_{r_{1}}^{r\left(\theta_{2}\right)} r_{2}{ }^{2} \mathrm{~d} r_{2} / r_{12} .
$$

In Eq. (11) the integration over $r_{2}$ is divided into these two ranges, since in the first range one must use Eq. (10a) and in the second (10b):

$$
V\left(r_{1}, \theta_{1}\right)=\iint \mathrm{d} \omega_{2} \sum_{k=0}^{\infty} P_{k}\left(\cos \theta_{12}\right) / r_{1}{ }^{k+1} \int_{0}^{r_{1}} r_{2}{ }^{k+2} \mathrm{~d} r_{2}+\iint \mathrm{d} \omega_{2} \sum_{k=0}^{\infty} r_{1}{ }^{k} P_{k}\left(\cos \theta_{12}\right) \int_{r_{1}}^{r\left(\theta_{2}\right)} \mathrm{d} r_{2} / r_{2}{ }^{k-1} .
$$

The integration over $\mathrm{d} r_{2}$ gives

$$
\begin{aligned}
V\left(r_{1}, \theta_{1}\right)=\sum_{k=0}^{\infty} r_{1}{ }^{2} /(k+3) & \iint \mathrm{d} \omega_{2} P_{k}\left(\cos \theta_{12}\right)+\sum_{k \neq 2} r_{1}^{k} /(-k+2) \iint \mathrm{d} \omega_{2} P_{k}\left(\cos \theta_{12}\right)\left(r\left(\theta_{2}\right)\right)^{-k+2} \\
& -\sum_{k \neq 2} r_{1}^{2} /(-k+2) \iint \mathrm{d} \omega_{2} P_{k}\left(\cos \theta_{12}\right)+\iint \mathrm{d} \omega_{2} P_{2}\left(\cos \theta_{12}\right) r_{1}{ }^{2} \ln \left(r\left(\theta_{2}\right) / r_{1}\right) .
\end{aligned}
$$

In Eq. (12), the first and third integrals may be evaluated by using the expansion

$$
P_{k}\left(\cos \theta_{12}\right)=\sum_{m=-k}^{+k} \frac{(k-m) !}{(k+m) !} P_{k^{m}}\left(\cos \theta_{1}\right) P_{k}^{m}\left(\cos \theta_{2}\right) e^{i m\left(\varphi_{1}-\varphi_{2}\right)} .
$$

The integration over $\phi_{2}$ gives a nonzero contribution only for $m=0$; and the integration over $\theta_{2}$ gives a nonzero contribution only for $k=0$. Thus

$$
\begin{aligned}
V\left(r_{1}, \theta_{1}\right)=(4 \pi / 3) r_{1}^{2}-2 \pi r_{1}^{2}+\sum_{k \neq 2} r_{1}^{k} /(-k+2) & \iint \mathrm{d} \omega_{2} P_{k}\left(\cos \theta_{12}\right) R^{-k+2}\left(1+\alpha_{0}+Q\left(\cos \theta_{2}\right)\right)^{-k+2} \\
& +\iint \mathrm{d} \omega_{2} P_{2}\left(\cos \theta_{12}\right) r_{1}^{2} \ln \left\{\left(R / r_{1}\right)\left(1+\alpha_{0}+Q\left(\cos \theta_{2}\right)\right)\right\} ;
\end{aligned}
$$




$$
\begin{aligned}
& =-(2 \pi / 3) r_{1}^{2}+R^{2} \sum_{k \neq 2}\left(r_{1} / R\right)^{k}\left(1+\alpha_{0}\right)^{-k+2} /(-k+2) \iint \mathrm{d} \omega_{2} P_{k}\left(\cos \theta_{12}\right) \\
& \quad \times\left\{1+\frac{(-k+2)}{1+\alpha_{0}} Q+\frac{(-k+2)(-k+1)}{2\left(1+\alpha_{0}\right)^{2}} Q^{2}+\cdots\right\} \\
& \left.\left.\quad+\iint \mathrm{d} \omega_{2} P_{2}\left(\cos \theta_{12}\right) r_{1}^{2}\right\} \ln \left(\left(1+\alpha_{0}\right) R / r_{1}\right)+\frac{Q}{1+\alpha_{0}}-\frac{Q^{2}}{2\left(1+\alpha_{0}\right)^{2}}+\cdots\right\} \\
& \approx-(2 \pi / 3) r_{1}{ }^{2}+R^{2} \sum_{k \neq 2}\left(r_{1} / R\right)^{k}\left(1+\alpha_{0}\right)^{-k+2} /(-k+2) \iint \mathrm{d} \omega_{2} P_{k}\left(\cos \theta_{12}\right) \\
& +\iint \mathrm{d} \omega_{2} P_{2}\left(\cos \theta_{12}\right) r_{1}^{2} \ln \left(\left(1+\alpha_{0}\right) R / r_{1}\right) \\
& +R^{2} \sum_{k=0}^{\infty}\left(r_{1} / R\right)^{k}\left(1+\alpha_{0}\right)^{-k+1} \iint \mathrm{d} \omega_{2} P_{k}\left(\cos \theta_{12}\right) Q\left(\cos \theta_{2}\right) \\
& +R^{2} \sum_{h=0}^{\infty}\left(r_{1} / R\right)^{k}\left(1+\alpha_{0}\right)^{-k}(-k+1) / 2 \iint \mathrm{d} \omega_{2} P_{k}\left(\cos \theta_{12}\right) Q^{2}\left(\cos \theta_{2}\right) .
\end{aligned}
$$

If one substitutes from Eq. (13) for $P_{k}\left(\cos \theta_{12}\right)$ in this last expression, it is evident that in all the integrals the only nonzero contribution from the integration over $\phi_{2}$ is for $m=0$; the first set of integrals is also nonzero only for $k=0$, and the second integral vanishes. The remaining integrals are of the form

$$
\begin{aligned}
I=2 \pi R^{2} \sum_{k=0}^{\infty}\left(r_{1} / R\right)^{k}\left(1+\alpha_{0}\right)^{-k+1} P_{k}\left(\cos \theta_{1}\right) \int_{0}^{\pi} \sin \theta_{2} \mathrm{~d} \theta_{2} P_{k}\left(\cos \theta_{2}\right) \sum_{n=1} \alpha_{n} P_{n}\left(\cos \theta_{2}\right) \\
I^{\prime}=\pi R^{2} \sum_{k=0}^{\infty}\left(r_{1} / R\right)^{k}\left(1+\alpha_{0}\right)^{-k}(-k+1) P_{k}\left(\cos \theta_{1}\right) \int_{0}^{\pi} \sin \theta_{2} \mathrm{~d} \theta_{2} P_{k}\left(\cos \theta_{2}\right) \\
\quad \times\left\{\sum_{n=1} \alpha_{n}{ }^{2} P_{n}^{2}\left(\cos \theta_{2}\right)+\sum_{n \neq m} \alpha_{n} \alpha_{m} P_{n}\left(\cos \theta_{2}\right) P_{m}\left(\cos \theta_{2}\right)\right\} .
\end{aligned}
$$

Thus

$$
I=4 \pi R^{2} \sum_{n=1}\left(r_{1} / R\right)^{n} \frac{\left(1+\alpha_{0}\right)^{-n+1}}{(2 n+1)} \alpha_{n} P_{n}\left(\cos \theta_{1}\right) ;
$$

and $I^{\prime}$ may be expressed in terms of the constants,

$$
c_{k n m}=\int_{0}^{\pi} \sin \theta_{2} \mathrm{~d} \theta_{2} P_{k}\left(\cos \theta_{2}\right) P_{n}\left(\cos \theta_{2}\right) P_{m}\left(\cos \theta_{2}\right), \quad \begin{array}{r}
k=0,1,2, \cdots \\
n, m=1,2, \cdots
\end{array}
$$

It may be noted that $c_{o n m}=0$ for $n \neq m$, and $c_{o n n}=2 /(2 n+1)$. In terms of the $c^{\prime}$,

$$
I^{\prime}=\pi R^{2} \sum_{k=0}^{\infty}\left(r_{1} / R\right)^{k}\left(1+\alpha_{0}\right)^{-k}(-k+1) P_{k}\left(\cos \theta_{1}\right)\left\{\sum_{n=1} \alpha_{n}^{2} c_{k n n}+\sum_{n \neq m} \alpha_{n} \alpha_{m} c_{k n m}\right\} .
$$

Upon collecting these results, one gets for $V\left(r_{1}, \theta_{1}\right)$,

$$
\begin{aligned}
V\left(r_{1}, \theta_{1}\right) \approx-(2 \pi / 3) r_{1}^{2} & +2 \pi R^{2}\left(1+\alpha_{0}\right)^{2}+4 \pi R^{2} \sum_{n=1}\left(r_{1} / R\right) \frac{\left(1+\alpha_{0}\right)^{-n+1}}{(2 n+1)} \alpha_{n} P_{n}\left(\cos \theta_{1}\right) \\
& +\pi R^{2} \sum_{k=0}^{\infty}\left(r_{1} / R\right)^{k}\left(1+\alpha_{0}\right)^{-k}(-k+1) P_{k}\left(\cos \theta_{1}\right)\left\{\sum_{n=1} \alpha_{n}^{2} c_{k n n}+\sum_{n \neq m} \alpha_{n} \alpha_{m} c_{l k n m}\right\}
\end{aligned}
$$


Integration of $V\left(r_{1}, \theta_{1}\right)$ over the volume of the deformed nucleus determines the total electrostatic energy. Terms containing $\alpha_{0}$ in powers higher than the first are dropped, as are terms containing the $\alpha_{n}$ 's in powers higher than the second. Thus, from the first term on the right-hand side of (14), one has

$$
-(2 \pi / 3) \int_{0}^{2 \pi} \mathrm{d} \varphi_{1} \int_{0}^{\pi} \sin \theta_{1} \mathrm{~d} \theta_{1} \int_{0}^{r\left(\theta_{1}\right)} r_{1}^{4} \mathrm{~d} r_{1} \approx-\left(8 \pi^{2} / 15\right) R^{5}(1+5 K) ;
$$

from the second term,

from the third term,

$$
2 \pi R^{2}\left(1+\alpha_{0}\right)^{2} \cdot 4 \pi R^{3} / 3 \approx 8 \pi^{2} R^{5}(1-2 K) / 3
$$

$$
4 \pi R^{5} \sum_{n=1} \frac{\alpha_{n}}{(2 n+1)} \frac{\left(1+\alpha_{0}\right)^{-n+1}}{(n+3)} \iint \mathrm{d} \omega_{2}\left(1+\alpha_{0}+Q\left(\cos \theta_{1}\right)\right)^{n+3} P_{n}\left(\cos \theta_{1}\right) \approx 16 \pi^{2} R^{5} \sum_{n=1} \alpha_{n}^{2} /(2 n+1)^{2} ;
$$

and from the fourth term,

$$
\begin{aligned}
\pi R^{5} \sum_{k=0}^{\infty}\left(1+\alpha_{0}\right)^{-k} \frac{(-k+1)}{(k+3)}\left\{\sum_{n=1} \alpha_{n}{ }^{2} c_{k n n}+\sum_{n \neq m} \alpha_{n} \alpha_{m} c_{k n m}\right\} \iint \mathrm{d} \omega_{1}\left(1+\alpha_{0}+Q\right)^{k+3} \cdot P_{k}\left(\cos \theta_{\mathrm{I}}\right) \\
\approx 4 \pi^{2} R^{5} / 3\left\{\sum_{n=1} \alpha_{n}^{2} c_{o n n}+\sum_{n \neq m} \alpha_{n} \alpha_{m} c_{o n m}\right\},
\end{aligned}
$$

since only the term $k=0$ contributes in the present approximation. Because of the values for $c_{o n m}$, this last term is equal to

$$
\left(8 \pi^{2} R^{5} / 3\right) \sum_{n=1} \alpha_{n}^{2} /(2 n+1)
$$

Addition of these terms gives

$$
E_{E} / \frac{1}{2} \rho^{2}=\left(16 \pi^{2} R^{5} / 3\right)\left\{2 / 5-\sum_{n=1} \alpha_{n}^{2} /(2 n+1)[1-3 /(2 n+1)]\right\} .
$$

The final result for the electrostatic energy in the present approximation may be expressed from Eq. (15) as

$$
E_{E}=3(Z e)^{2} / 5 R\left\{1-5 \sum_{n=1} \alpha_{n}{ }^{2}(n-1) /(2 n+1)^{2}\right\} .
$$

It may be noted that $\alpha_{1}$ makes no contribution either to $E_{E}$ or to $E_{S}$; this result is to be expected since the term $\alpha_{1} P_{1}(\cos \theta)$ in Eq. (2) corresponds approximately to a translation of the nucleus without deformation.

It is now possible to discuss nuclear stability conditions from Eqs. (6) and (16). In order that a nucleus be stable for small deformations, the potential energy difference $E_{S}+E_{E}-4 \pi R^{2} O-(3 / 5)(Z e)^{2} / R$ $=\Omega$ should be positive. Now

$$
\Omega=\sum_{n=2} \alpha_{n}^{2}(n-1) /(2 n+1)\left\{4 \pi R^{2} O(n+2) / 2-\left[3(Z e)^{2} / 5 R\right] 5 /(2 n+1)\right\} .
$$

It is clear from Eq. (17) that the stability limit is lower for the deformation characterized by $n=2$ than for any other; hence one may assume that the term with $n=2$ gives the critical stability limit:

$$
4 \pi R^{2} O \cdot 2-(3 / 5)(Z e)^{2} / R>0 ;
$$

or, using the relation $R=r_{0} A^{\frac{1}{3}}$, that

$$
Z^{2} / A<2 \cdot 4 \pi r_{0}^{2} O /(3 / 5)\left(e^{2} / r_{0}\right) \text {. }
$$

The right-hand side of Eq. (19) may be regarded as giving the limiting value for $Z^{2} / A$; if the values for $r_{0}$ and $O$ given in the preceding section are used,

$$
\left(Z^{2} / A\right)_{1 \text { im }}=2 \cdot 4 \pi r_{0}^{2} O /(3 / 5)\left(e^{2} / r_{0}\right) \approx 47.5 \text {. }
$$

The largest values of $Z^{2} / A$ occur in the heaviest nuclei, but even for these, $Z^{2} / A$ is less than the limiting value. For ${ }_{92} \mathrm{U}^{235}, Z^{2} / A=36.0$; for 
${ }_{92} \mathrm{U}^{238}, Z^{2} / A=35.6$; for ${ }_{90} \mathrm{Th}^{232}, Z^{2} / A=34.9$. All the nuclei are thus stable for small deformations, and spontaneous fission cannot be expected to take place.

While the potential energy function $\Omega\left(\alpha_{2}, \alpha_{3}\right.$, $\cdots$ ) has a minimum at $\alpha_{2}=\alpha_{3}=\cdots=0$, it has other minimums for a sufficiently heavy nucleus at other values of the $\alpha$ 's corresponding to a configuration in which the nucleus is divided into two fragments. These minimums are much lower than $\Omega(0,0, \cdots)$, as was shown in Sect. 2 where the large energy release following fission was discussed. In principle, it is possible to calculate $\Omega\left(\alpha_{2}, \alpha_{3}, \cdots\right)$ for large as well as small values of the $\alpha$ 's, and such a calculation would determine the course of the function $\Omega$ between the spherical undisturbed state and a state of division. Although approximate determinations of $\Omega$ have been made for large deformations, ${ }^{6,11}$ the discussion here will be only qualitative. If one pictures a series of deformations with amplitudes increasing toward, say, a dumbbell shape, it is clear that the potential energy function $\Omega$ will increase until some critical deformation is reached in which there is an instability; that is, the potential energy would be decreased either by approaching the configuration of division or by a return to the neighborhood of the spherical shape. In this critical configuration the work required for a small deformation in either "direction" vanishes in the first order. There is thus on the potential energy hypersurface, $\Omega\left(\alpha_{2}, \alpha_{3}, \cdots\right)$, a smooth-topped ridge between the valley at $\alpha_{2}=\alpha_{3}=\cdots=0$, and a valley at some configuration $\alpha_{2}=\alpha_{2}^{\prime}, \alpha_{3}=\alpha_{3}^{\prime}$, etc., corresponding to a divided nucleus (Fig. 2). The lowest point on such a ridge determines the minimum energy input necessary to arrive at the divided configuration. If this configuration occurs for $\alpha_{2}=\alpha_{2}{ }^{f}$, $\alpha_{3}=\alpha_{3}{ }^{f}$, etc., this minimum energy increment will be $E_{f}=\Omega\left(\alpha_{2}{ }^{f}, \alpha_{3}{ }^{f}, \cdots\right) . E_{f}$ is the characteristic critical energy for the fission process and will be a function of the ratio. $\left(Z^{2} / A\right) /\left(Z^{2} / A\right)_{1 \text { im }}=x$; as $x$ approaches unity, $E_{f}(x)$ will decrease monotonically toward zero.

A simple possible mechanism for getting excitation in a heavy nucleus over the critical limit $E_{f}(x)$ is neutron capture. In uranium it was

${ }^{11}$ R. D. Present and J. K. Knipp, Phys. Rev. 57, 751 (1940); 57, 1188 (1940).

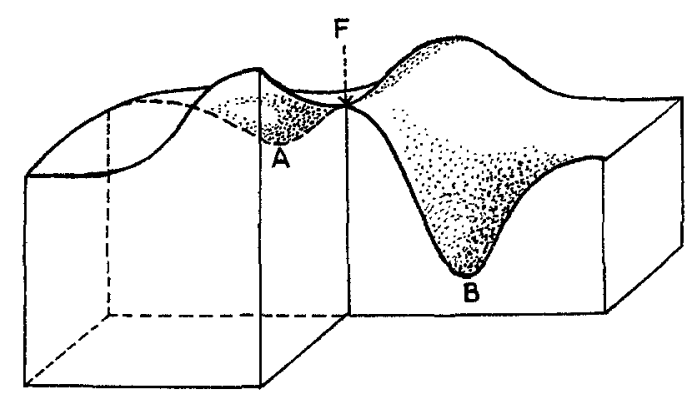

Frg. 2. Diagrammatic sketch of the potential energy surface, illustrating the potential barrier between the configuration $A$, which corresponds to the undistorted spherical shape of the nucleus, and configuration $B$, which corresponds to a state of division into two fragments. The point $F$ indicates the saddle point on the barrier between $A$ and $B$.

found experimentally that fission was produced by the capture of neutrons of negligible energy (neutrons of thermal energy $\sim 0.02 \mathrm{ev}$ ), and also by the capture of neutrons of high energy. Uranium has three isotopes, ${ }_{92} \mathrm{U}^{234},{ }_{92} \mathrm{U}^{235}$ and ${ }_{92} \mathrm{U}^{238}$, with these abundance ratios: $:^{12} \mathrm{U}^{234} / \mathrm{U}^{288}$ $=1 / 17,000 ; U^{235} / \mathrm{U}^{238}=1 / 139$. Since the isotope ${ }_{92} \mathrm{U}^{234}$ is present in such a small amount, it cannot be expected to contribute appreciably to the fission reactions. In a theoretical analysis of the experimental situation, it was predicted by Bohr ${ }^{5}$ that the fission in uranium with thermal neutrons came from neutron capture by the isotope ${ }_{92} \mathrm{U}^{235}$ and that the important contribution to the fission with energetic neutrons came from neutron capture by ${ }_{92} \mathrm{U}^{238}$. This theoretical analysis was confirmed by experiments with small samples of the separated isotopes..$^{13}$ It was pointed out in Sect. 2 that the slow neutron capture reactions,

$$
\begin{aligned}
& { }_{92} \mathrm{U}^{235}+{ }_{0} n^{1} \rightarrow{ }_{92} \mathrm{U}^{236}, \\
& { }_{92} \mathrm{U}^{238}+{ }_{0} n^{1} \rightarrow{ }_{92} \mathrm{U}^{239},
\end{aligned}
$$

give an excitation energy in ${ }_{92} \mathrm{U}^{236}$ of about 6.4 Mev, while in ${ }_{92} \mathrm{U}^{239}$ the excitation is about 5.2 Mev. It therefore appears that ${ }_{92} \mathrm{U}^{236}$, for which $x=0.75_{5}$, has $E_{f}<6.4 \mathrm{Mev}$. It also appears that ${ }_{92} \mathrm{U}^{239}$, for which $x=0.74_{6}$, has $E_{f}>5.2 \mathrm{Mev}$. The minimum neutron bombarding energy that gives fission in ${ }_{92} \mathrm{U}^{239}$ has been measured ${ }^{14}$ to be 0.35

12 A. O. Nier, Phys. Rev. 55, 150 (1939).

${ }^{13}$ Nier, Booth, Dunning and Grosse, Phys. Rev. 57, 748 (1940); Kingdon, Pollock, Booth and Dunning, Phys. Rev, 57, 749 (1940).

${ }_{14}$ Haxby, Shoupp, Stephens and Wells, Phys. Rev. 58, 199 (1940). 
$\mathrm{Mev} \pm 0.10 \mathrm{Mev}$. It follows that $E_{f}$ for ${ }_{92} \mathrm{U}^{239}$ is approximately $5.5 \mathrm{Mev}$. Fission has also been observed when thorium is bombarded with sufficiently energetic neutrons. When the thorium isotope ${ }_{90} \mathrm{Th}^{232}$ captures a slow neutron, the excitation energy is estimated at 5.2 Mev. ${ }^{6}$ The threshold neutron energy in thorium has been measured $^{15}$ to be $1.1 \pm 0.10 \mathrm{Mev}$. Thus $E_{f}$ for ${ }_{90} \mathrm{Th}^{233}$ is approximately $6.3 \mathrm{Mev}$; the corresponding value of $x$ is 0.73 .

\section{4.}

The preceding considerations have referred only to the potential energy changes in nuclear deformations. One may also readily calculate the kinetic energy of the oscillations accompanying these deformations. For an incompressible fluid in irrotational motion, the velocity $\mathrm{v}$ at any point in the fluid is derivable from a potential, $\mathrm{v}=\operatorname{grad} \psi$. The velocity potential $\psi$ satisfies the Laplace equation, $\Delta \psi=0$. The mass density in the nucleus is constant, and, if it is denoted by $d$, the total kinetic energy will be

$$
T=\frac{1}{2} d \iint_{V} \int_{(\operatorname{grad} \psi)^{2} \mathrm{~d} \tau .}
$$

From the identity, $(\operatorname{grad} \psi)^{2} \equiv \operatorname{div}(\psi \operatorname{grad} \psi)$ $-\psi \Delta \psi=\operatorname{div}(\psi \operatorname{grad} \psi)$, one sees that the volume integral may be transformed into a surface integral over the nuclear boundary. For small oscillations, the boundary may be approximated by the undeformed surface, that is, the surface of a sphere of radius $R$. Thus

$$
T \approx \frac{1}{2} d R^{2} \int_{0}^{2 \pi} \int_{0}^{\pi} \psi(\partial \psi / \partial r)_{r=R} \sin \theta \mathrm{d} \theta \mathrm{d} \varphi
$$

It is clear that for deformations with zonal symmetry $\psi$ will be expressible as a sum of zonal harmonics,

$\psi=\beta_{0}+\beta_{1} r P_{1}(\cos \theta)+\beta_{2} r^{2} P_{2}(\cos \theta)$

and

$$
+\cdots+\beta_{n} r^{n} P_{n}(\cos \theta)+\cdots ;
$$

$$
\partial \psi / \partial r=\sum_{n=0} n \beta_{n} r^{n-1} P_{n}(\cos \theta) \text {. }
$$

${ }^{15}$ Haxby, Shoupp, Stephens and Wells, Phys. Rev. 57, $1088(1940)$.
Therefore,

$$
\begin{aligned}
T & =\frac{1}{2} d \sum_{m, n} n R^{n+m+1} \beta_{n} \beta_{m} \int_{0}^{2 \pi} \int_{0}^{\pi} P_{m} P_{n} \sin \theta \mathrm{d} \theta, \\
& =2 \pi d \sum_{n} n /(2 n+1) R^{2 n+1} \beta_{n}{ }^{2} .
\end{aligned}
$$

Now

$$
r=R\left\{1+\alpha_{0}+\sum_{n=2} \alpha_{n} P_{n}(\cos \theta)\right\}
$$

so that, in the first approximation in the $\alpha$ 's, the radial velocity $\dot{r}[\equiv \mathrm{d} r / \mathrm{d} t]$ is given by

$$
\dot{r}=R \sum_{n=2} \dot{\alpha}_{n} P_{n}(\cos \theta)
$$

A comparison of Eqs. (23) and (22a) determines the unknown coefficients $\beta_{n}: \beta_{n}=\dot{\alpha}_{n} / n R^{n-2}$; and the kinetic energy becomes

$$
T=2 \pi d R^{5} \sum_{n=2} \dot{\alpha}_{n}{ }^{2} / n(2 n+1) .
$$

If one neglects the small difference between the masses of the proton and neutron and writes $M_{n} \approx M_{p}=M$, then $d=M A /\left(4 \pi R^{3} / 3\right)$ and

$$
\begin{aligned}
T & =(3 / 2) M A R^{2} \sum_{n=2} \dot{\alpha}_{n}^{2} / n(2 n+1) \\
& =(3 / 2) M A^{5 / 3} r_{0}^{2} \sum_{n=2} \dot{\alpha}_{n^{2}}{ }^{2} / n(2 n+1) .
\end{aligned}
$$

From Eqs. (17) and (24) one sees that, in the present approximation, there is no coupling between the modes of oscillation; the total energy is a sum of the form

$$
\sum_{n=2}\left(T_{n}+\Omega_{n}\right) \text {. }
$$

Each mode of oscillation has the energy form of a simple harmonic oscillator. If momentums $p_{n}$ canonically conjugate to the generalized coordinates $\alpha_{n}$ are introduced,

$$
p_{n}=\partial T / \partial \dot{\alpha}_{n}=3 M A^{5 / 3} r_{0}^{2} \dot{\alpha}_{n} / n(2 n+1),
$$

the energy of each mode may then be expressed in Hamiltonian form,

$$
H_{n}=\left(1 / 2 m_{n}\right) p_{n}{ }^{2}+\left(k_{n}{ }^{2} / 2\right) \alpha_{n}{ }^{2} .
$$

From Eq. (24),

$$
m_{n}=\frac{3}{n(2 n+1)} M A^{5 / 3} r_{0}{ }^{2},
$$

and from (Eq. 17),

$$
k_{n}=\frac{2(n-1)}{(2 n+1)} 4 \pi r_{0}^{2} A^{\frac{2}{3}} O\{(n+2) / 2-10 x /(2 n+1)\},
$$


where, as before, $x=\left(Z^{2} / A\right) /\left(Z^{2} / A\right)_{\text {1im. }}$. The angular frequency $\omega_{n}$ of the $n$th mode of oscillation is $\left(k_{n} / m_{n}\right)^{\frac{1}{2}}$.

It should be pointed out that the calculations given here are similar to those carried out by Rayleigh in his study of the oscillations of water droplets in jets. ${ }^{16}$ The only essential difference between the calculations for the nuclear problem and those for the water droplet is that an electrostatic charge on a droplet is distributed uniformly over the surface, whereas for the nucleus it is distributed uniformly through the volume. There is also for charged water droplets a stability limit, which determines the fineness of the jet.

It may be of interest to remark that the potential energy of deformation and the kinetic energy of oscillation may be calculated in a somewhat different way from that presented in Secs. 3 and 4 . One need only determine the electrostatic potential at the surface in the first approximation in the $\alpha_{n}$ 's. The pressure at the surface is also calculated in the first approximation from the expression $p=O\left(1 / R_{1}+1 / R_{2}\right)$. The total curvature $\left(1 / R_{1}+1 / R_{2}\right)$ is readily found. ${ }^{17}$ From the expressions for the pressure and the electrostatic potential at the surface, the change in potential energy due to deformation is determined. The frequencies of oscillation are found from the hydrodynamical relation $p / d=-\partial \psi / \partial t-V(r(\cos \theta), \theta)+$ const. These calculations are carried out as readily for distortions ex-

${ }^{16}$ Theory of Sound, ed. 2, Vol. II, \$364; Phil. Mag. 14, 184 (1882).

${ }_{17}$ Lamb, Hydrodynamics, ed. 6, \$275. pressed in terms of surface harmonics as for those limited to zonal symmetry.

So far the discussion of nuclear stability and fission has been purely classical, and the classical description must be justified by consideration of the quantum aspects of the problem. It is clear from Eq. (25) that each mode of oscillation has the quantum-mechanical behavior of a simple harmonic oscillator with its characteristic zeropoint energy and zero-point oscillations. In order to describe classically deformations of the order of magnitude of nuclear dimensions, the amplitude of the zero-point oscillations must be appreciably smaller than the nuclear radius. If the mean square amplitude of the zero-point oscillation for the $n$th mode is denoted by $\left\langle\alpha_{n}^{2}\right\rangle_{\text {ero point }}$, then one finds readily that

$$
\left\langle\alpha_{n}^{2}\right\rangle_{\text {zero point }}=\hbar / 2\left(m_{n} k_{n}\right)^{\frac{2}{2}},
$$

which is indeed small compared with $R^{2}$. The zero-point energy is $E_{n}=\left(\frac{1}{2}\right) \hbar \omega_{n}=\left(\frac{1}{2}\right) \hbar\left(k_{n} / m_{n}\right)^{\frac{1}{2}}$; in particular, for $n=2$, one finds in uranium $E_{2} \sim 0.4 \mathrm{Mev}$. This zero-point energy is small compared with the energy" $E_{f}$ which is about 5 Mev. Hence, one may also describe the critical deformations in the neighborhood of $\alpha_{n}=\alpha_{n}{ }^{f}$ that lead to fission, in a classical way.

\title{
Presentation of the Concept of Liquid Structure
}

\author{
C. D. Thomas, Missouri School of Mines and Metallurgy, Rolla, Missouri
}

AND

N. S. GINGRICH, University of Missouri, Columbia, Missouri

\begin{abstract}
A LTHOUGH it is an easy matter to present to students the structure of an idealized crystal with its regularly arranged atoms and it is not a difficult task to visualize the complete lack of structure in a gas, the description of the arrangement of atoms in a liquid has suffered because it is an intermediate case. This lack of adequate description for the "structure" of a liquid has been alleviated by recent work on the diffraction of $x$-rays by liquids. Since $x$-rays have served very effectively in investigating the arrangements of atoms in crystals and the distributions of atoms in monatomic gases, it is reasonable to look to them for a description of the
\end{abstract}

arrangement of atoms in a liquid. X-ray diffraction studies lead to the determination of the distribution of atoms about a given atom (for an element) in the solid, liquid or gaseous state, and this atomic distribution curve is characteristic of the state of the material. Hence the atomic distribution curve can be used to present the concept of the "structure" of matter in any state. In the case of liquids, this, perhaps, is the most quantitative expression of "structure" that can be given.

To illustrate the idea of atomic distribution, consider a regular square array of atoms in a plane as shown in Fig. 1. Assuming an ideal 